\title{
Regulatory potential of post-translational modifications in bacteria
}

\author{
Christophe Grangeasse ${ }^{1}$, Jörg Stülke ${ }^{2}$ and Ivan Mijakovic ${ }^{3 *}$ \\ ${ }^{1}$ Bases Moléculaires et Structurales des Systèmes Infectieux, UMR 5086, Centre National de la Recherche Scientifique, \\ University of Lyon, Lyon, France, ${ }^{2}$ Department of General Microbiology, Institute for Microbiology and Genetics, University of \\ Göttingen, Göttingen, Germany, ${ }^{3}$ Systems and Synthetic Biology Division, Department of Biology and Biological Engineering, \\ Chalmers University of Technology, Göteborg, Sweden
}

Keywords: phosphorylation, S-thiolation, dehydration, hydroxylation, $\mathrm{N}$-glycosylation, protein kinases, antimicrobial peptides, proteomics

Bacteria are often viewed as simple organisms, with very basic and robust cellular regulation, optimized for rapid growth. While they certainly fit that description, bacteria also possess an amazing capacity for adaptation, and diversity of survival strategies, including variations of cell morphology, size, mode of growth and developmental behavior. Post-translational modifications (PTMs) of proteins contribute significantly to bacterial adaptability and cell cycle control. Research on PTMs in bacteria started with the assumption that they lack many features regularly found in more complex organisms. However, ongoing investigation keeps revealing new types of PTMs of bacterial proteins. This research topic gathers a number of articles discussing the advanced methods for systematic study of bacterial PTMs, approaches to utilize PTMs for biotechnological purposes, and revealing new cellular functions controlled by PTMs.

Most bacterial PTMs are dynamic and reversible. This allows the cell to exploit them as regulatory devices. It also means that the full understanding of the cellular roles of different PTMs necessitates global, quantitative and time-resolved studies. One seminal paper in this topic reports a novel proteomics approach for such quantitative studies: the Intensity Based Absolute Quantitation (iBAQ). Using this approach, the authors have quantified the expression and the occupancy of various PTM sites in the proteome of Escherichia coli (Soufi et al., 2015). The authors report remarkable differences in expression and occupancy of PTMs sites under different growth conditions. The dataset comprised 2300 proteins, which is close to $90 \%$ of the expressed proteome. This is an important landmark, since proteomics in general has not yet attained the same level of coverage that global transcriptome studies can achieve.

Among different bacterial PTMs, protein phosphorylation is the most extensively studied one, and in this topic it features prominently. Several papers present global studies of protein phosphorylation in bacteria (Spät et al., 2015), including important bacterial pathogens (Fortuin et al., 2015; Nakedi et al., 2015). The focus on pathogens is understandable, since protein phosphorylation, and several other PTMs, are heavily involved in different infection strategies displayed by bacterial pathogens (Michard and Doublet, 2015). In addition to phosphoproteome studies, interactomics is also featured as a useful approach to chart the phosphorylationbased regulatory networks. It enables researchers to trace the connections among protein kinases, phosphatases, and their substrates (Shi et al., 2014a). This approach for reconstructing phosphorylation networks highlights the capacity of bacterial proteins kinases from different families to interact with, and phosphorylate, each other (Shi et al., 2014b). Protein-tyrosine kinases, and their various roles in bacterial physiology were in the focus of several review and opinion articles in this topic (Barák, 2014; Gerwig and Stülke, 2014; Mijakovic and Deutscher, 2015). 
Other topic contributions highlight the broad spectrum of PTMs involved in key cellular processes. In particular, novel modifications are discussed: redox regulation via reversible Sthiolation (Loi et al., 2015), post-translational hydroxylation (van Staalduinen and Jia, 2015) and the role of citrullination for the interaction between bacteria and human mucosal surfaces (Sofat et al., 2015). Several experimental papers report studies on post-translationally modified antimicrobial peptides known as lantibiotics. These are ribosomally synthesized peptides which can efficiently inhibit the growth of Gram-positive bacteria. A study by Zhou et al. (2015) described an engineering strategy to make the lantibiotics more effective in inhibiting several important bacterial pathogens. In another study, Khusainov et al. (2015) describe the active site of the nisin dehydratase. This enzyme, essential for the production of the lantibiotic nisin, converts serines, and threonines, to dehydroalanine and dehydrobutyrine residues, respectively. Interestingly, bacterial PTM systems, such as the $\mathrm{N}$-glycosylation machinery, are also being exploited and engineered to facilitate the production of recombinant vaccines (Garcia-Quintanilla et al., 2014). Another focal point of the topic are the PTMs targeting the PII proteins,

\section{References}

Barák, I. (2014). Complexity of bacterial phosphorylation interaction network. Front. Microbiol. 5:725. doi: 10.3389/fmicb.2014.00725

Fortuin, S., Tomazella, G. G., Nagaraj, N., Sampson, S. L., Gey van Pittius, N. C., Soares, N. C., et al. (2015). Phosphoproteomics analysis of a clinical Mycobacterium tuberculosis Beijing isolate: expanding the mycobacterial phosphoproteome catalog. Front. Microbiol. 6:6. doi: 10.3389/fmicb.2015.00006

Garcia-Quintanilla, F., Iwashkiw, J. A., Price, N. L., Stratilo, C., and Feldman, M. F. (2014). Production of a recombinant vaccine candidate against Burkholderia pseudomallei exploiting the bacterial $\mathrm{N}$-glycosylation machinery. Front. Microbiol. 5:381. doi: 10.3389/fmicb.2014.00381

Gerwig, J., and Stülke, J. (2014). Far from being well understood: multiple protein phosphorylation events control cell differentiation in Bacillus subtilis at different levels. Front. Microbiol. 5:704. doi: 10.3389/fmicb.2014.00704

Khusainov, R., van Heel, A. J., Lubelski, J., Moll, G. N., and Kuipers, O. P. (2015). Identification of essential amino acid residues in the nisin dehydratase NisB. Front. Microbiol. 6:102. doi: 10.3389/fmicb.2015.00102

Loi, V. V., Rossius, M., and Antelmann, H. (2015). Redox regulation by reversible protein S-thiolation in bacteria. Front. Microbiol. 6:187. doi: 10.3389/fmicb.2015.00187

Merrick, M. (2015). Post-translational modification of P II signal transduction proteins. Front. Microbiol. 5:763. doi: 10.3389/fmicb.2014.00763

Michard, C., and Doublet, P. (2015). Post-translational modifications are key players of the Legionella pneumophila infection strategy. Front. Microbiol. 6:87. doi: $10.3389 /$ fmicb.2015.00087

Mijakovic, I., and Deutscher, J. (2015). Protein-tyrosine phosphorylation in Bacillus subtilis: a 10-year retrospective. Front. Microbiol. 6:18. doi: 10.3389/fmicb.2015.00018

Nakedi, K. C., Nel, A. J. M., Garnett, S., Blackburn, J. M., and Soares, N. C. (2015). Comparative Ser/Thr/Tyr phosphoproteomics between two mycobacterial species: the fast growing Mycobacterium smegmatis and the slow growing Mycobacterium bovis BCG. Front. Microbiol. 6:237. doi: 10.3389/fmicb.2015.00237

Radchenko, M. V., Thornton, J., and Merrick, M. (2014). Association and dissociation of the GlnK-AmtB complex in response to cellular nitrogen status can occur in the absence of GlnK post-translational modification. Front. Microbiol. 5:731. doi: 10.3389/fmicb.2014.00731

Shi, L., Pigeonneau, N., Ravikumar, V., Dobrinic, P., Macek, B., Franjevic, D., et al. (2014b). Cross-phosphorylation of bacterial serine/threonine and which are the key signal transduction proteins involved in the control of nitrogen metabolism in bacteria and archaea (Radchenko et al., 2014; Merrick, 2015). Finally, Stannek et al. (2015) contributed a study on a regulatory mechanism involving arginine phosphorylation and regulated proteolysis.

In conclusion, the contributions in this topic reflect the diversity of bacterial PTMs. Several studies highlight one important emergent feature of PTM systems: their capacity to interact with each other, creating an additional level of complexity in the cellular regulation. This is one of the key features of bacterial PTM systems and a challenge which future studies will have to address.

\section{Acknowledgments}

This topic was organized to accompany the First International Conference on Post-translational Modifications in Bacteria, held in Göttingen (Germany), 9-10 September 2014. We would like to take this opportunity to thank all the conference participants, and especially those who contributed their work to this topic.

tyrosine protein kinases on key regulatory residues. Front. Microbiol. 5:495. doi: 10.3389/fmicb.2014.00495

Shi, L., Pigeonneau, N., Ventroux, M., Derouiche, A., Bidnenko, V., Mijakovic, I., et al. (2014a). Protein-tyrosine phosphorylation interaction network in Bacillus subtilis reveals new substrates, kinase activators and kinase cross-talk. Front. Microbiol. 5:538. doi: 10.3389/fmicb.2014. 00538

Sofat, N., Wait, R., Robertson, S. D., Baines, D. L., and Baker, E. H. (2015). Interaction between extracellular matrix molecules and microbial pathogens: evidence for the missing link in autoimmunity with rheumatoid arthritis as a disease model. Front. Microbiol. 5:783. doi: 10.3389/fmicb.2014. 00783

Soufi, B., Krug, K., Harst, A., and Macek, B. (2015). Characterization of the E. coli proteome and its modifications during growth and ethanol stress. Front. Microbiol. 6:103. doi: 10.3389/fmicb.2015.00103

Spät, P., Macek, B., and Forchhammer, K. (2015). Phosphoproteome of the cyanobacterium Synechocystis sp. PCC 6803 and its dynamics during nitrogen starvation. Front. Microbiol. 6:248. doi: 10.3389/fmicb.2015.00248

Stannek, L., Gunka, K., Care, R. A., Gerth, U., and Commichau, F. M. (2015). Factors that mediate and prevent degradation of the inactive and unstable GudB protein in Bacillus subtilis. Front. Microbiol. 5:758. doi: 10.3389/fmicb. 2014.00758

Zhou, L., van Heel, A. J., and Kuipers, O. P. (2015). The length of a lantibiotic hinge region has profound influence on antimicrobial activity and host specificity. Front. Microbiol. 6:11. doi: 10.3389/fmicb.2015.00011

van Staalduinen, L. M., and Jia, Z. (2015). Post-translational hydroxylation by $2 \mathrm{OG} / \mathrm{Fe}$ (II)-dependent oxygenases as a novel regulatory mechanism in bacteria. Front. Microbiol. 5:798. doi: 10.3389/fmicb.2014.00798

Conflict of Interest Statement: The authors declare that the research was conducted in the absence of any commercial or financial relationships that could be construed as a potential conflict of interest.

Copyright (C) 2015 Grangeasse, Stülke and Mijakovic. This is an open-access article distributed under the terms of the Creative Commons Attribution License (CC $B Y)$. The use, distribution or reproduction in other forums is permitted, provided the original author(s) or licensor are credited and that the original publication in this journal is cited, in accordance with accepted academic practice. No use, distribution or reproduction is permitted which does not comply with these terms. 